\title{
Decentralized Event-based Scheduling for Shared-Resource Networked Control Systems
}

\author{
Mohammad H. Mamduhi ${ }^{1}$, Domagoj Tolić ${ }^{2}$ and Sandra Hirche ${ }^{1}$
}

\begin{abstract}
In this paper, we introduce a decentralized eventtriggered scheduling scheme for multi-loop Networked Control Systems (NCSs) in which the individual control loops are coupled through a shared communication channel. The proposed scheduling design combines deterministic and probabilistic attributes to efficiently allocate the limited communication resource among the control loops in an event-based fashion. Based on local error thresholds, each control loop determines whether to compete for the channel access. As a result, control loops with higher transmission priorities are more likely to utilize the channel, which in turn leads to more efficient usage of the limited resource. Each eligible subsystem then attempts to transmit at times specified by local waiting times, which are randomly distributed and local-error dependent. In this manner, the probability of data collisions in the communication channel is reduced. If the channel capacity is reached at some time instants, data packets are dropped. We demonstrate stochastic stability of such NCSs in terms of Lyapunov Stability in Probability (LSP). The numerical results show that our approach improves resource utilization and reduces the networked-induced error variance in comparison with timetriggered, random access, and centralized scheduling policies.
\end{abstract}

\section{INTRODUCTION}

Traditional digital control systems are characterized by collocated sensors, actuators and controllers as well as by time-triggered control schemes with periodic sampling. With the advent of new technologies, the parts of control systems are becoming evermore spatially distributed and their interaction is being increasingly supported by shared communication networks, which usually impose energy and capacity limitations [1]. In order to utilize the limited communication and energy resources more efficiently, event-triggered control and scheduling schemes have been proposed recently [2]-[7].

These references suggest that it is often more beneficial to transmit the sampled data upon the occurrence of certain events rather than at periodic time instants. This is even more so in case of large-scale systems due to the sheer amount of exchanged data. While time-triggered access schemes usually offer lower complexity as they are offline, event-based rules excel in efficient allocation and robustness.

In the event-based paradigm, events are typically triggered by either deterministic [8], [9], or stochastic policies [10][13]. Deterministic policies usually have better performance

\footnotetext{
${ }^{1}$ M. H. Mamduhi and S. Hirche are with the Institute for Information-oriented Control, Technische Universität München, Arcisstraße 21, D-80290 München, Germany; http://www.itr.ei.tum.de, \{mh. mamduhi, hirche\}@tum. de

${ }^{2}$ Domagoj Tolic is with the University of Zagreb, Faculty of Electrical Engineering and Computing, Unska 3, 10000 Zagreb, Croatia. His work is supported by the European Community Seventh Framework Program under grant No. 285939 (ACROSS). domagoj.tolicefer.hr
}

as they award the channel to the entity with the highest priority. As a basic deterministic protocol the Try-OnceDiscard (TOD) awards the medium access to the system with the largest estimation error [9]. However, TOD is prone to noise and can cope with collisions only with a given predefined priority order, and hence is not convenient for practical realizations, e.g. wireless multihop networks [14].

The Maximal Allowable Transfer Interval (MATI) plays a crucial role while analyzing stability of systems with deterministic schedules by deriving the upper bound on the time interval between two successive transmissions [8], [9]. On the contrary, MATI does not apply to stochastic schemes as the intervals between consecutive transmissions usually cannot be upper bounded uniformly with probability one. This calls for new stability approaches for stochastic NCSs [10]-[13]. Probabilistic policies are more suitable to deal with stochastic systems with model uncertainties and channel imperfections [10], [12], [13], [15]. While stability analysis for single-loop NCSs are well addressed, see e.g., [11], [12], less investigations are carried out for multi-loop NCSs, with [10], [13] being notable exceptions. Altogether, applicable event-based scheduling designs for multi-loop NCSs that efficiently utilize the limited resource are certainly of interest.

In this paper, we introduce a decentralized bi-character scheduling policy for a multiple-loop NCS sharing a communication network with limited capacity. We show that our approach preserves stability of the overall NCS while utilizing the limited resources more efficiently. In our scheme, the order of transmissions is determined locally. First, the subsystems with local errors not exceeding pre-specified local thresholds do not compete for channel access in favor of those with larger errors. The larger the error threshold of a subsystem is, the larger the error it can tolerate between two sampling periods becomes. The subsystems with errors larger than their corresponding thresholds compete for the channel access in a probabilistic fashion. The bigger the local error of a subsystem is, the higher its chance of transmission becomes. The probabilistic feature of our protocol facilitates the consideration of unbounded disturbances and collisions without a predefined prioritization. Adopting an emulative control design, we show stability of the resulting multi-loop NCS in terms of Lyapunov stability in probability (LSP).

In the remainder of this paper, Section II states the problem formulation and introduces our decentralized scheduling law. In Section III, we analyze stability of the overall NCS. Simulation results are illustrated in Section IV.

Notation In this paper, the conditional expectation is denoted $\mathrm{E}[\cdot \mid \cdot]$. The Gaussian distribution with mean $\mu$ and covariance 


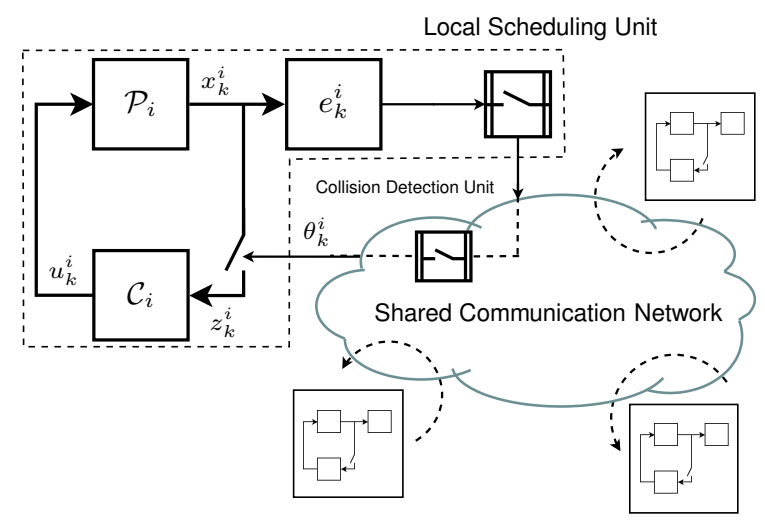

Fig. 1. A multi-loop NCS with a shared communication channel and local scheduling mechanism.

matrix $X$ is represented by $\mathcal{N}(\mu, X)$, while $\mathrm{P}[\mathcal{A}]$ denotes the probability that event $\mathcal{A}$ occurs. A vector with superscript $i$ belongs to control loop $i$, while the subscript $k$ denotes the time step. For matrices, subscript $i$ indicates the belonging subsystem while superscript $n$ denotes the matrix power.

\section{PROBLEM STATEMENT}

Consider an NCS composed of $N$ heterogeneous LTI control loops coupled through a shared communication medium, as schematically depicted in Fig. 1. Each control loop consists of a stochastic plant $\mathcal{P}_{i}$, and a feedback controller $\mathcal{C}_{i}$. The plant $\mathcal{P}_{i}$ is modeled by the stochastic difference equation

$$
x_{k+1}^{i}=A_{i} x_{k}^{i}+B_{i} u_{k}^{i}+w_{k}^{i},
$$

where the disturbance process $w_{k}^{i} \in \mathbb{R}^{n_{i}}$ is i.i.d. with $\mathcal{N}(0, I)$ at each time $k$, and $A_{i} \in \mathbb{R}^{n_{i} \times n_{i}}, B_{i} \in \mathbb{R}^{n_{i} \times m_{i}}$ describe system and input matrices, respectively. Considering unity covariance Gaussian distribution for disturbance process is merely for notational convenience. Since our results are independent of initial state, $x_{0}^{i}$ is allowed to be any random variable with arbitrary finite variance distributions. At each time-step $k$, the binary scheduling variable $\delta_{k}^{i} \in\{0,1\}$ denotes if a subsystem $i$ is awarded the channel access, i.e.

$$
\delta_{k}^{i}= \begin{cases}1, & x_{k}^{i} \text { is sent for transmission } \\ 0, & x_{k}^{i} \text { is blocked. }\end{cases}
$$

Each loop is assumed to be driven by a local state feedback controller updated at every time step $k$ either by the true state $x_{k}^{i}$ (in case $\delta_{k}^{i}=1$ ) or by the estimated state $\hat{x}_{k}^{i}$ (in case $\left.\delta_{k}^{i}=0\right)$. Essentially, we consider control laws $\gamma^{i}$, given by

$$
u_{k}^{i}=\gamma^{i}\left(Z_{k}^{i}\right)=-L_{i} \mathrm{E}\left[x_{k}^{i} \mid Z_{k}^{i}\right],
$$

where $Z_{k}^{i}=\left\{x_{0}^{i}, \delta_{0}^{i} \ldots, x_{k}^{i}, \delta_{k}^{i}\right\}$ is the update history and $L_{i}$ is any stabilizing feedback gain. Notice that, in accordance with the emulation-based frameworks, the synthesis of $L_{i}$ 's is not explicitly addressed here. Knowing that the plants are stabilized in the absence of a communication network, we focus on the scheduling process. In case $\delta_{k}^{i}=0$, a Kalmanlike estimator with local knowledge $Z_{k-1}^{i}$ computes $\hat{x}_{k}^{i}$ as

$$
\mathrm{E}\left[x_{k}^{i} \mid Z_{k}^{i}\right]=\left(A_{i}-B_{i} L_{i}\right) \mathrm{E}\left[x_{k-1}^{i} \mid Z_{k-1}^{i}\right],
$$

with initial distribution $\mathrm{E}\left[x_{0}^{i} \mid Z_{0}^{i}\right]=0$. The estimate in (3) is well behaved since a stabilizing gain $L_{i}$ ensures that the closed-loop matrix $\left(A_{i}-B_{i} L_{i}\right)$ is Hurwitz. The local network-induced error is defined as $e_{k}^{i}=x_{k}^{i}-\mathrm{E}\left[x_{k}^{i} \mid Z_{k}^{i}\right]$ for each subsystem $i \in\{1 \ldots, N\}$, and evolves as

$$
e_{k+1}^{i}=\left(1-\delta_{k+1}^{i}\right) A_{i} e_{k}^{i}+w_{k}^{i},
$$

where the sequence of decisions within one time period is

$$
\cdots \rightarrow e_{k} \rightarrow \delta_{k+1} \rightarrow z_{k+1} \rightarrow u_{k+1} \rightarrow e_{k+1} \rightarrow \cdots
$$

Having (1)-(4), the aggregate networked state $\left(x_{k}^{i}, e_{k}^{i}\right)$ has triangular dynamics within each local control loop as

$$
\left[\begin{array}{c}
x_{k+1}^{i} \\
e_{k+1}^{i}
\end{array}\right]=\left[\begin{array}{cc}
A_{i}-B_{i} L_{i} & \left(1-\delta_{k+1}^{i}\right) B_{i} L_{i} \\
0 & \left(1-\delta_{k+1}^{i}\right) A_{i}
\end{array}\right]\left[\begin{array}{c}
x_{k}^{i} \\
e_{k}^{i}
\end{array}\right]+\left[\begin{array}{c}
w_{k}^{i} \\
w_{k}^{i}
\end{array}\right] \text {. }
$$

This implies that the evolution of the error sate $e_{k}^{i}$ is independent of the system state $x_{k}^{i}$.

\section{A. Decentralized Scheduling Mechanism}

We assume that the operational time scale of the communication channel is much finer than that of the local control systems, i.e. between each two time steps $k \rightarrow k+1$, the communication time slots are $\{k h, k h+\tau, \ldots, k h+(h-$ 1) $\tau,(k+1) h\}$, with $\tau$ a fixed time duration and the integer $h$ denoting the number of samples within one control period. Assume $h \gg 1$. We aim for a decentralized approach, i.e. the $i^{\text {th }}$ subsystem is provided with only local information $A_{i}, B_{i}, W_{i}, \lambda_{i}, Z_{k}^{i}$ and the distribution of $x_{0}^{i}$, where $\lambda_{i}$ is the local error threshold. Thus, every subsystem $i$ knows its latest local error $e_{k^{\prime}}^{i}$ as well as $\lambda_{i}$ to decide whether to compete for channel access at time $k^{\prime}+1$. If the weighted squared norm of its latest error is smaller or equal to the given local threshold, subsystem $i$ will be excluded from the channel access competition. This process represents the deterministic feature of the scheduling design. Thus, we have

$$
\mathrm{P}\left[\delta_{k^{\prime}+1}^{i}=1 \mid e_{k^{\prime}}^{i}\right]=0 \quad \text { if } \quad\left\|e_{k^{\prime}}^{i}\right\|_{Q_{i}}^{2} \leq \lambda_{i},
$$

where $\left\|e_{k}^{i}\right\|_{Q_{i}}^{2}:=e_{k}^{i^{\top}} Q_{i} e_{k}^{i}$ and $Q_{i}$ is a positive definite matrix.

Each subsystem $i$, eligible to compete for the channel access at time step $k^{\prime}+1$, satisfies the following condition

$$
\left\|e_{k^{\prime}}^{i}\right\|_{Q_{i}}^{2}>\lambda_{i}
$$

A decentralized probabilistic mechanism allocates the channel among the qualified subsystems such that the ones with larger errors have higher chances of channel access. In the absence of centralized information, our probabilistic mechanism determines the transmission order locally within each subsystem. For simplicity, we assume that only one subsystem can transmit at multiples of $\tau$ at each time-step $k$

$$
\sum_{i=1}^{N} \delta_{k}^{i}=1
$$

The provided results can be extended for $\sum_{i=1}^{N} \delta_{k}^{i}=c<N$.

We introduce integer random variables $\nu_{k^{\prime}}^{i} \in$ $\{\tau, 2 \tau, \ldots,(h-1) \tau\}$, called waiting times, which denote the time a transmission-eligible subsystem $i$ waits before listening to the channel, i.e. at time $k^{\prime} h+\nu_{k^{\prime}}^{i}$. If the 
channel is free, subsystem $i$ transmits. Otherwise, it backs off and does not attempt to transmit over $\left\{k^{\prime} h, k^{\prime} h+\tau, \ldots, k^{\prime} h+(h-1) \tau,\left(k^{\prime}+1\right) h\right\}$ again. We propose, for a subsystem $i$ which satisfies (6), that the waiting time $\nu_{k^{\prime}}^{i}$ is chosen randomly from a finite-variance concave probability mass function with the error-dependent mean

$$
\mathrm{E}\left[\nu_{k^{\prime}}^{i}\right]=\frac{1}{\left\|e_{k^{\prime}}^{i}\right\|_{Q_{i}}^{2}} .
$$

Assumption (6) ensures $\mathrm{E}\left[\nu_{k^{\prime}}^{i}\right] \in\left(0, \lambda_{i}^{-1}\right)$. The random variables $\nu_{k^{\prime}}^{i}$ are chosen according to their corresponding local probability mass functions. The concavity of the local distribution emphasizes the prioritized character as it ensures the random waiting times are chosen with higher probabilities around the mean (8). As our policy is decentralized, the possibility of collisions should also be accounted for. A collision occurs if at least two subsystems choose exactly identical waiting times. Each subsystem is informed by the collision detection unit, in case a collision occurs. At every time step $k$, a successful transmission is reported via $\gamma_{k}$ as

$$
\gamma_{k}^{i}= \begin{cases}1, & x_{k}^{i} \text { successfully received } \\ 0, & x_{k}^{i} \text { dropped. }\end{cases}
$$

Therefore, the dynamics of the error state $e_{k}^{i}$ becomes

$$
e_{k+1}^{i}=\left(1-\theta_{k+1}^{i}\right) A_{i} e_{k}^{i}+w_{k}^{i},
$$

where $\theta_{k}^{i}=\delta_{k}^{i} \gamma_{k}^{i}$. Principally, by choosing the waiting times randomly the probability of collision is reduced by introducing the communication sub-frames as a collision avoidance mechanism, and the transmission chance for subsystems with high errors is increased via the choice of local distributions. We can achieve such a design within a TCP-like protocol, where the acknowledgment of a successful transmission is sent over an error-free reverse link to each subsystem.

Let $\mathcal{G}_{k^{\prime}}$ denote the set of eligible subsystems for transmission at time-step $k^{\prime}+1$. Thus, considering (7), the probability that subsystem $j \in \mathcal{G}_{k^{\prime}}$ transmits at time-step $k^{\prime}+1$ is

$$
\mathrm{P}\left[\delta_{k^{\prime}+1}^{j}=1 \mid e_{k}^{j}\right]=\mathrm{P}\left[\nu_{k^{\prime}}^{j}<\nu_{k^{\prime}}^{l}\right], \quad \forall l \in \mathcal{G}_{k^{\prime}}, l \neq j .
$$

Basically, a subsystem $j$ starts listening the channel according to its chosen waiting time. If the channel is free, it transmits which ensures its waiting time has been shorter than all other eligible subsystems $l \in \mathcal{G}_{k^{\prime}}, l \neq j$, i.e. $\nu_{k^{\prime}}^{j}<\nu_{k^{\prime}}^{l}$. The subsystems that find the channel occupied, might try to transmit in future time-steps adhering to similar procedure, if they are eligible depending on their updated error values. Fig. 2 depicts our proposed access scheme, schematically.

Remark 1: The dependence of random waiting times, which is a MAC layer parameter, on error values from the application (i.e. the control) layer, couples the medium access to the application. This is in line with recent trends of crosslayer design - in contrast to the classical separation of OSI layers in classical communication protocols.

We define the aggregate error state $e_{k} \in \mathbb{R}^{n}$ by stacking the error vectors of different subsystems as follows:

$$
e_{k}=\left(e_{k}^{1^{\top}}, \ldots, e_{k}^{N^{\top}}\right)^{\top}
$$

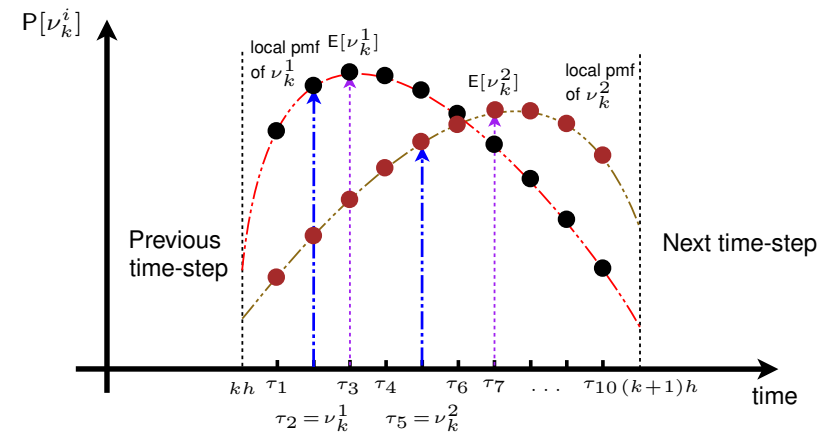

Fig. 2. Two subsystems compete for channel access by randomly choosing their waiting times $\nu_{k}^{1}$ and $\nu_{k}^{2}$, according to their corresponding local probability mass functions with error-dependent means $\mathrm{E}\left[\nu_{k}^{1}\right]$ and $\mathrm{E}\left[\nu_{k}^{2}\right]$.

where $n=\sum_{i=1}^{N} n_{i}$. The introduced scheduling policy, is a randomized policy depending only on the most recent error values. Since the scheduler restarts at the beginning of each sample time, regardless of which subsystem has previously transmitted, the scheduling variable depends only on the latest error value. Although the error evolution is affected by the history of error state and scheduling variables, the randomization in channel allocation renders the scheduling variables statistically independent from their previous values. Therefore, (11) possesses the Markov property. Moreover, as the difference equation in (4) is time-invariant and the process $w_{k}^{i}$ is i.i.d. for $i \in\{1, \ldots, N\}$, the Markov chain (11) is homogeneous. Moreover, as the disturbance distribution is absolutely continuous with a positive density function, the Markov chain is aperiodic and $\psi$-irreducible.

\section{STABILITY ANALYSIS}

In this section, we study stability of multi-loop NCSs with shared communication networks subject to the capacity constraint (7), and the proposed decentralized scheduling policy in (5) and (10). Essentially, we show Lyapunov stability in probability (LSP) for the overall NCS of interest. Before proceeding, we state some preliminaries about LSP.

\section{A. Preliminaries}

Definition 1: (Lyapunov Stability in Probability (LSP), [16]) A linear system with state vector $x_{k}$ possesses LSP if given $\varepsilon, \varepsilon^{\prime}>0$, exists $\rho\left(\varepsilon, \varepsilon^{\prime}\right)>0$ such that $\left|x_{0}\right|<\rho$ implies

$$
\lim _{k \rightarrow \infty} \sup \mathrm{P}\left[x_{k}^{\top} x_{k} \geq \varepsilon^{\prime}\right] \leq \varepsilon .
$$

The following lemma shows the LSP is achievable by solely considering the error state $e_{k}$.

Lemma 1: For an NCS described by (1)-(4), the condition in (12) is equivalent to

$$
\lim _{k \rightarrow \infty} \sup \mathrm{P}\left[e_{k}^{\top} e_{k} \geq \xi^{\prime}\right] \leq \xi
$$

where $\xi^{\prime}>0$ and the constant $\xi$ fulfills $0 \leq \xi \leq \varepsilon$.

Proof: The system state $x_{k}^{i}$ for each loop $i$ evolves as

$$
x_{k+1}^{i}=\left(A_{i}-B_{i} L_{i}\right) x_{k}^{i}+\left(1-\theta_{k+1}^{i}\right) B_{i} L_{i} e_{k}^{i}+w_{k}^{i} .
$$

As already discussed, the evolution of the error $e_{k}^{i}$ is independent of the system state $x_{k}^{i}$ within each individual control 
loop. Furthermore, by assumption the emulative control law (2) ensures the closed-loop matrix $\left(A_{i}-B_{i} L_{i}\right)$ is Hurwitz. Together with the assumption that $x_{0}^{i}$ has a bounded variance distribution, it follows that the system state $x_{k}^{i}$ is converging. In addition, the disturbance process $w_{k}^{i}$ is i.i.d. according to $\mathcal{N}(0, I)$, and is bounded in probability. Thus, showing $\lim _{k \rightarrow \infty} \sup \mathrm{P}\left[e_{k}^{i^{\top}} e_{k}^{i} \geq \xi_{i}^{\prime}\right] \leq \xi_{i}$ ensures existence of constants $\varepsilon_{i}$ and $\varepsilon_{i}^{\prime}>0$ such that $\lim _{k \rightarrow \infty} \sup \mathrm{P}\left[x_{k}^{i^{\top}} x_{k}^{i} \geq \varepsilon_{i}^{\prime}\right] \leq$ $\varepsilon_{i}$. As individual loops operate independently, we take the aggregate NCS state $\left(x_{k}, e_{k}\right)$. Then, the existence of $\xi$ and $\xi^{\prime}>0$ such that $\lim _{k \rightarrow \infty} \sup \mathrm{P}\left[e_{k}^{\top} e_{k} \geq \xi^{\prime}\right] \leq \xi$, implies existence of $\varepsilon$ and $\varepsilon^{\prime}>0$ such that $\lim _{k \rightarrow \infty} \sup \mathrm{P}\left[x_{k}^{\top} x_{k} \geq \varepsilon^{\prime}\right] \leq$ $\varepsilon$, and the proof readily follows.

As expected values are more straightforward in pursuing further analysis than probabilities, it holds from the Markov's inequality that for $\xi^{\prime}>0$

$$
\mathrm{P}\left[e_{k}^{\mathrm{T}} e_{k} \geq \xi^{\prime}\right] \leq \frac{\mathrm{E}\left[e_{k}^{\top} e_{k}\right]}{\xi^{\prime}} .
$$

This confirms that showing the error is uniformly bounded in expectation ensures finding appropriate $\xi$ and $\xi^{\prime}>0$ such that (13) is satisfied for arbitrary $\rho\left(\xi^{\prime}, \xi\right)$. Introducing positive definite matrices $Q_{i}$, we focus on deriving an upper bound for the expectation of weighted quadratic error norm

$$
\mathrm{E}\left[e_{k}^{\top} Q e_{k}\right]=\sum_{i=1}^{N} \mathrm{E}\left[e_{k}^{i^{\top}} Q_{i} e_{k}^{i}\right]=\sum_{i=1}^{N} \mathrm{E}\left[\left\|e_{k}^{i}\right\|_{Q_{i}}^{2}\right],
$$

where $Q=\operatorname{diag}\left(Q_{i}\right)$. This modifies the condition (13) as

$$
\lim _{k \rightarrow \infty} \sup \mathrm{P}\left[e_{k}^{\top} Q e_{k} \geq \bar{\xi}^{\prime}\right] \leq \bar{\xi}
$$

Due to the capacity constraint (7), the boundedness of (16) cannot always be shown over one step transition. This observation is shown via the following illustrative example: Illustrative example Let an NCS be composed of two identical scalar subsystems competing for the sole channel slot. For simplicity, assume $Q_{1}=Q_{2}=1, \lambda_{1}=\lambda_{2}=\bar{\lambda}$ and $e_{k}^{1}=e_{k}^{2}=\bar{e}_{k}$. In addition, consider that the condition (6) is fulfilled, and that both systems have identical distributions to choose their waiting times $\nu_{k+1}^{1}$ and $\nu_{k+1}^{2}$. According to (8), the distributions have the same means $\frac{1}{\left\|\bar{e}_{k}\right\|_{2}^{2}}$. Thus, the transmission chance for each system is $\frac{1}{2}$. We have from (4)

$$
\begin{aligned}
& \sum_{i=1,2} \mathrm{E}\left[\left\|e_{k+1}^{i}\right\|_{I}^{2}\right]=\sum_{i=1,2} \mathrm{E}\left[\left\|\left(1-\theta_{k+1}^{i}\right) A_{i} e_{k}^{i}+w_{k}^{i}\right\|_{I}^{2}\right] \\
& =\frac{1}{2}\left(\mathrm{E}\left[\left\|A \bar{e}_{k}+w_{k}^{1}\right\|_{I}^{2} \mid e_{k}\right]+\mathrm{E}\left[\left\|w_{k}^{2}\right\|_{I}^{2}\right]\right) \\
& +\frac{1}{2}\left(\mathrm{E}\left[\left\|A \bar{e}_{k}+w_{k}^{2}\right\|_{I}^{2} \mid e_{k}\right]+\mathrm{E}\left[\left\|w_{k}^{1}\right\|_{I}^{2}\right]\right)=2+\left\|A \bar{e}_{k}\right\|_{I}^{2},
\end{aligned}
$$

which is not bounded for arbitrary $\bar{e}_{k}$ and system matrix $A$. Intuitively, between two consecutive transmissions of each subsystem, they operate in open loop. Hence, in general, the respective local errors are expected to grow. Thus to obtain boundedness of error state, all subsystems need to have transmission chances. Due to the constraint (7), one infers that an interval of length $N$ provides enough transmission possibilities. (see [17] for a comprehensive discussion).

\section{B. Stability Analysis - Lyapunov Stability in Probability}

To show LSP, let the NCS of interest operate over the interval $[k, k+N]$, with arbitrary initial state $e_{k}$. We assume that the NCS freely operates from the initial time $k$ until $k+N-1$ and we predict the error evolution considering all the possible scenarios under the introduced scheduling policy over the interval $[k, k+N-1]$. Then, looking at time-step $k+N$, we show the aggregate error state $e_{k+N}$ fulfills (16). We divide the subsystems $i \in\{1, \ldots, N\}$ at each time-step $k^{\prime} \in[k, k+N-1]$ into two disjoint sets as

$$
i \in \begin{cases}\mathcal{G}_{k^{\prime}} & \left\|e_{k^{\prime}}^{i}\right\|_{Q_{i}}^{2}>\lambda_{i} \\ \overline{\mathcal{G}}_{k^{\prime}} & \left\|e_{k^{\prime}}^{i}\right\|_{Q_{i}}^{2} \leq \lambda_{i},\end{cases}
$$

where $\mathcal{G}_{k^{\prime}} \cup \overline{\mathcal{G}}_{k^{\prime}}=N$. According to (5), subsystems belonging to $\mathcal{G}_{k^{\prime}}$ are considered for transmission at time $k^{\prime}+1$. Note that not only a transmission results in error decrement for a subsystem, but the disturbance process might also decrease the error. Therefore, the inclusion in either set $\mathcal{G}_{k^{\prime}}$ or $\overline{\mathcal{G}}_{k^{\prime}}$ depends on both transmission occurrence and disturbance $w_{k^{\prime}}^{i}$. To take this into account, we discern three complementary and mutually exclusive cases, covering the entire state space the Markov chain $e_{k}$ evolves, at time-step $k+N-1$ as:

Subsystem $i$ :

$c_{1}$ : has either successfully transmitted or not within the past $N-1$ time-steps, and is in set $\overline{\mathcal{G}}_{k+N-1}$, i.e.

$$
i \in \overline{\mathcal{G}}_{k+N-1} \Rightarrow\left\|e_{k+N-1}^{i}\right\|_{Q_{i}}^{2} \leq \lambda_{i}
$$

$c_{2}$ : has successfully transmitted at least once within the past $N-1$ time-steps, and is in set $\mathcal{G}_{k+N-1}$, i.e. $\exists k^{\prime} \in[k, k+N-1]: \theta_{k^{\prime}}^{i}=1$ and $\left\|e_{k+N-1}^{i}\right\|_{Q_{i}}^{2}>\lambda_{i}$,

$c_{3}$ : has not successfully transmitted within the past $N-1$ time-steps, and is in set $\mathcal{G}_{k+N-1}$, i.e.

$$
\forall k^{\prime} \in[k, k+N-1]: \theta_{k^{\prime}}^{i}=0 \text { and }\left\|e_{k+N-1}^{i}\right\|_{Q_{i}}^{2}>\lambda_{i} .
$$

Theorem 1: Consider an NCS with $N$ heterogeneous LTI control loops, with the plants given by (1), sharing a communication channel subject to the constraint (7). Given the control law (2) and scheduling laws (5) and (10), the NCS of interest is Lyapunov stable in probability.

Proof: We study the boundedness of error expectation over the interval $[k, k+N]$ for cases $c_{1}-c_{3}$. Since, the cases are complementary and mutually exclusive, we can calculate the probability for each case to happen, and express (16) as

$$
\sum_{i=1}^{N} \mathrm{E}\left[\left\|e_{k+N}^{i}\right\|_{Q_{i}}^{i}\right]=\sum_{c_{l}} \mathrm{P}_{c_{l}} \mathrm{E}\left[\left\|e_{k+N}^{i}\right\|_{Q_{i}}^{i} \mid c_{l}, \mathrm{P}_{c_{l}}\right]
$$

where $\sum_{l=1}^{3} \mathrm{P}_{c_{l}}=1$.

Suppose that some subsystems $i$ belong to $c_{1}$. Since $i \in$ $\overline{\mathcal{G}}_{k+N-1}$, it follows from (18) that $\left\|e_{k+N-1}^{i}\right\|_{Q_{i}}^{2} \leq \lambda_{i}$. Thus, they are not eligible for transmission at time $k+N$, i.e. $\delta_{k+N}^{i}=0$. Then, it follows from (4) and (19)

$$
\begin{aligned}
\sum_{i \in c_{1}} \mathrm{E} & {\left[\left\|e_{k+N}^{i}\right\|_{Q_{i}}^{2} \mid e_{k}\right]=\sum_{i \in c_{1}} \mathrm{E}\left[\left\|A_{i} e_{k+N-1}^{i}+w_{k+N-1}^{i}\right\|_{Q_{i}}^{2} \mid e_{k}\right] } \\
& \leq \sum_{c_{1}}\left\|A_{i}\right\|_{2}^{2} \mathrm{E}\left[\left\|e_{k+N-1}^{i}\right\|_{Q_{i}}^{2} \mid e_{k}\right]+\mathrm{E}\left[\left\|w_{k+N-1}^{i}\right\|_{Q_{i}}^{2}\right] \\
& \leq \sum_{c_{1}}\left\|A_{i}\right\|_{2}^{2} \lambda_{i}+\mathrm{E}\left[\left\|w_{k+N-1}^{i}\right\|_{Q_{i}}^{2}\right] .
\end{aligned}
$$


This fulfills the condition (17) with $\bar{\xi}^{\prime}>\sum_{c_{1}}\left\|A_{i}\right\|_{2}^{2} \lambda_{i}+$ $\mathrm{E}\left[\left\|w_{k+N-1}^{i}\right\|_{Q_{i}}^{2}\right]$, and $\bar{\xi}=\frac{\sum_{c_{1}} \mathrm{E}\left[\left\|e_{k+N}^{i}\right\|_{Q_{i}}^{2} \mid e_{k}\right]}{\bar{\xi}^{\prime}}<1$.

For some $i \in c_{2}$, let a successful transmission is occurred at time-step $k+r_{i}^{\prime}$, where $r_{i}^{\prime} \in[1, N-1]$, i.e. $\theta_{k+r_{i}^{\prime}}^{i}=1$. We express $e_{k+N}^{i}$ as a function of the error at time $k+r_{i}^{\prime}-1$ as

$$
\begin{aligned}
e_{k+N}^{i} & =\prod_{j=r_{i}^{\prime}}^{N}\left(1-\theta_{k+j}^{i}\right) A_{i}^{N-r_{i}^{\prime}+1} e_{k+r_{i}^{\prime}-1}^{i} \\
& +\sum_{r=r_{i}^{\prime}}^{N}\left[\prod_{j=r+1}^{N}\left(1-\theta_{k+j}^{i}\right) A_{i}^{N-r} w_{k+r-1}^{i}\right],
\end{aligned}
$$

where we define $\prod_{N+1}^{N}\left(1-\theta_{k+j}^{i}\right):=1$. The first term of the above equality vanishes as $\theta_{k+r_{i}^{\prime}}^{i}=1$. By statistical independence of $w_{k+r-1}^{i}$ and $\theta_{k+j}^{i}$, it follows from (21)

$$
\begin{aligned}
& \sum_{c_{2}} \mathrm{E}\left[\left\|e_{k+N}^{i}\right\|_{Q_{i}}^{2} \mid e_{k}\right] \\
& =\sum_{c_{2}} \mathrm{E}\left[\left\|\sum_{r=r_{i}^{\prime}}^{N} \prod_{j=r+1}^{N}\left[1-\theta_{k+j}^{i}\right] A_{i}^{N-r} w_{k+r-1}^{i}\right\|_{Q_{i}}^{2}\right] \\
& \leq \sum_{c_{2}} \sum_{r=r_{i}^{\prime}}^{N} \mathrm{E}\left[\left\|A_{i}^{N-r} w_{k+r-1}^{i}\right\|_{Q_{i}}^{2}\right]
\end{aligned}
$$

In fact, we disregard the scheduling process in the last inequality. We are allowed to do so since $\prod_{j=r+1}^{N}\left[1-\theta_{k+j}^{i}\right] \leq$ 1. Hence, the condition (17) is satisfied considering $\bar{\xi}^{\prime}$ chosen to be larger than (22), and $\bar{\xi}=\frac{\sum_{c_{2}} \mathrm{E}\left[\left\|e_{k+N}^{i}\right\|_{Q_{i}}^{2} \mid e_{k}\right]}{\xi^{\prime}}<1$.

The subsystems in the third case are eligible for channel access at time $k+N$. To infer (19), we split the third case $c_{3}$ into two complementary and disjoint sub-cases as follows:

$l_{1}^{c_{3}} \quad$ system $i$ has not transmitted within the past $N-1$ time steps, but has been in $\overline{\mathcal{G}}$ at least once, the last occurred at a time $k+r_{i}^{\prime}$, with $r_{i}^{\prime} \in[0, \ldots, N-2]$,

$l_{2}^{c 3}$ system $i$ has not transmitted within the past $N-1$ timesteps, and has been in $\mathcal{G}$ for all time $[k, k+N-1]$.

Recall that the subsystems $i \in c_{3}$ belong to $\mathcal{G}_{k+N-1}$. For sub-case $l_{1}^{c_{3}}, k+r_{i}^{\prime}$ is the last time for which $i \in \overline{\mathcal{G}}_{k+r_{i}^{\prime}}$, which in turn implies $\left\|e_{k+r_{i}^{\prime}}^{i}\right\|_{Q_{i}}^{2} \leq \lambda_{i}$. Knowing that $\theta_{k^{\prime}}^{i}=0$ for $i \in c_{3}$ until time-step $k+N$, we reach

$$
\begin{aligned}
& \sum_{l_{1}^{c_{3}}} \mathrm{E}\left[\left\|e_{k+N}^{i}\right\|_{Q_{i}}^{2} \mid e_{k}\right] \leq \\
& \sum_{l_{1}^{c_{3}}}\left[\left\|A_{i}^{N-r_{i}^{\prime}}\right\|_{2}^{2} \lambda_{i}+\sum_{r=r_{i}^{\prime}}^{N-1} \mathrm{E}\left[\left\|A_{i}^{N-r-1} w_{k+r}^{i}\right\|_{Q_{i}}^{2}\right]\right] .
\end{aligned}
$$

The condition (17) is met by choosing $\bar{\xi}^{\prime}$ larger than the uniform upper bound (23), and $\bar{\xi}=\frac{\sum_{l_{1} c_{3}} \mathrm{E}\left[\left\|e_{k+N}^{i}\right\|_{Q_{i}}^{2} \mid e_{k}\right]}{\xi^{\prime}}<1$.

The subsystems $j \in l_{2}^{c_{3}}$ have always been candidates for channel access, i.e. $j \in \mathcal{G}_{[k, k+N-1]}$. Hence, $\left\|e_{k^{\prime}}^{j}\right\|_{Q_{j}}^{2}>\lambda_{j}$ for all $k^{\prime} \in[k, k+N-1]$. From (4), we conclude

$$
\begin{aligned}
& \sum_{l_{2}^{c_{3}}} \mathrm{E}\left[\left\|e_{k+N}^{j}\right\|_{Q_{j}}^{2} \mid e_{k}\right] \\
& \leq \sum_{l_{2}^{c_{3}}} \mathrm{E}\left[\left\|A_{j} e_{k+N-1}^{j}\right\|_{Q_{j}}^{2} \mid e_{k}\right]+\mathrm{E}\left[\left\|w_{k+N-1}^{j}\right\|_{Q_{j}}^{2}\right] .
\end{aligned}
$$

Expression (24) is not uniformly bounded since the term $e_{k+N-1}^{j}$ in (24) is not bounded according to (18). However, as the considered cases cannot happen all together, we calculate the probability for sub-case $l_{2}^{c_{3}}$ to happen according to the scheduling policy (10). Note that in deriving (20), (22) and (23) it was not necessary to consider the probability of happening the corresponding cases. To calculate the probability of happening we need to consider the possibility of collisions. As collisions may happen at all time steps, there is a non-zero probability that all the scheduled packets collide. This means all subsystems operate in open-loop at all time steps. We investigate two collision scenarios; 1) there has been at least one successful transmission over the interval $[k, k+N-1]$, and 2) there has been no successful transmission over the entire interval. We assume if a collision is reported, then the channel is not awarded to any subsystem.

Investigating the first scenario, we assume that whenever a collision is detected and consequently all the subsystems have to operate open loop, a virtual control loop has successfully transmitted instead of a real subsystem. This means at the time the collision occurs, $N$ real subsystems and one virtual one share the communication channel and channel is awarded to the virtual subsystem. The virtual loops have the same discrete LTI dynamics as in (1). As the worst case situation, let the channel experiences $m<N-1$ collisions in the interval $[k, k+N-1]$. Thus, at time $k+N$ we have $N$ real and $m$ virtual subsystems, where all virtual ones have transmitted. Since, we have $N+m$ subsystems, we need to extend our interval to $\left[k, k_{m}\right]$, where $k_{m}=k+N+m$. Consideration of virtual loops is merely to justify the longer interval and plays no more role in the analysis. If a subsystem $j \in l_{2}^{c_{3}}$ transmits at $k_{m}$, then its error becomes bounded in expectation. Otherwise, if $j$ has never transmitted, then there exists another subsystem, say $i$, which has transmitted more than once. Let $k+\bar{r}$ denote the most recent step in which $\theta_{k+\bar{r}}^{i}=1$ for $\bar{r} \leq N+m-1$. The probability that subsystem $i$ re-transmits at the final time step $k_{m}$, in the presence of the subsystem $j \in l_{2}^{c_{3}}$ can be expressed as

$$
\begin{aligned}
& \mathrm{P}\left[\theta_{k_{m}}^{i}=1 \mid \theta_{k+\bar{r}}^{i}=1, \theta_{k^{\prime}}^{j}=0 \quad \forall k^{\prime} \in\left[k, k_{m}\right]\right] \\
& \quad=\mathrm{P}\left[\nu_{k_{m}-1}^{j}>\nu_{k_{m}-1}^{i} \mid \theta_{k+\bar{r}}^{i}=1, \theta_{k^{\prime}}^{j}=0 \quad \forall k^{\prime} \in\left[k, k_{m}\right]\right] \\
& \quad \leq \frac{\mathrm{E}\left[\nu_{k_{m}-1}^{j} \mid \theta_{k^{\prime}}^{j}=0 \quad \forall k^{\prime} \in\left[k, k_{m}\right]\right]}{s_{k_{m}-1}^{i} \tau}
\end{aligned}
$$

where, the last expression follows from Markov's inequality considering the positive constant waiting time $\nu_{k_{m}-1}^{i}=$ $s_{k_{m}-1}^{i} \tau$ corresponds to subsystem $i$ is given, and $s_{k_{m}-1}^{i} \in$ $\{1, \ldots, h-1\}$. The latest error value of subsystem $j$ at time $k_{m}-1$ is required to be given in order to have an expectation of the waiting times $\nu_{k_{m}-1}^{j}$. Therefore, having the last expression conditioned on $e_{k_{m}-1}^{j}$, we have from the law of iterated expectation that

$$
\begin{aligned}
& \frac{\mathrm{E}\left[\nu_{k_{m}-1}^{j} \mid \theta_{k^{\prime}}^{j}=0 \quad \forall k^{\prime} \in\left[k, k_{m}\right]\right]}{s_{k_{m}-1}^{i} \tau} \\
& =\frac{\mathrm{E}\left[\mathrm{E}\left[\nu_{k_{m}-1}^{j} \mid e_{k_{m}-1}^{j}\right] \mid \theta_{k^{\prime}}^{j}=0 \quad \forall k^{\prime} \in\left[k, k_{m}\right]\right]}{s_{k_{m}-1}^{i} \tau} \\
& =\frac{1}{s_{k_{m}-1}^{i} \tau\left\|e_{k_{m}-1}^{j}\right\|_{Q_{j}}^{j}}
\end{aligned}
$$


where the last equality follows from (8). The expression (25) confirms that having large error values correspond to subsystems $j \in l_{2}^{c_{3}}$ with no prior transmission reduces the probability of re-transmission of a subsystem $i \notin l_{2}^{c_{3}}$.

Having (19) extended for the interval $\left[k, k_{m}\right]$ and considering the expression (24) for the expectation of error for a subsystem $j \in l_{2}^{c_{3}}$, we employ (25) as follows

$$
\begin{aligned}
& \sum_{l_{2}^{c_{3}}} \mathrm{P}_{l_{2}^{c_{3}}} \mathrm{E}\left[\left\|e_{k_{m}}^{j}\right\|_{Q_{j}}^{2} \mid e_{k}\right] \\
& =\sum_{l_{2}^{c_{3}}} \mathrm{P}\left[\nu_{k_{m}-1}^{j}>\nu_{k_{m}-1}^{i}\right] \mathrm{E}\left[\left\|e_{k_{m}}^{j}\right\|_{Q_{j}}^{2} \mid e_{k}\right] \\
& \leq \sum_{l_{2}^{c_{3}}} \frac{\left\|A_{j}\right\|_{2}^{2}\left\|e_{k_{m}-1}^{j}\right\|_{Q_{j}}^{2}}{s_{k_{m}-1}^{i} \tau\left\|e_{k_{m}-1}^{j}\right\|_{Q_{j}}^{2}}+\frac{\mathrm{E}\left[\left\|w_{k_{m}-1}^{j}\right\|_{Q_{j}}^{2}\right]}{s_{k_{m}-1}^{i} \tau\left\|e_{k_{m}-1}^{j}\right\|_{Q_{j}}^{2}} \\
& \leq \sum_{l_{2}^{c_{3}}} \frac{\left\|A_{j}\right\|_{2}^{2}}{s_{k_{m}-1}^{i} \tau}+\frac{\mathrm{E}\left[\left\|w_{k_{m}-1}^{j}\right\|_{Q_{j}}^{2}\right]}{\lambda_{j} s_{k_{m}-1}^{i} \tau},
\end{aligned}
$$

where the last inequality follows from knowing that $\left\|e_{k_{m}-1}^{j}\right\|_{Q_{j}}^{2}>\lambda_{j}$ for every subsystem $j \in l_{2}^{c_{3}}$. Since (26) is uniformly bounded, (17) holds by selecting $\bar{\xi}=$ $\underline{\sum_{l_{2} c_{3}} \mathrm{E}\left[\left\|e_{k_{m}}^{j}\right\|_{Q_{j}}^{2} \mid e_{k}\right]}$

$\frac{\xi^{\prime}}{\text { interval }}<1$ and $\bar{\xi}^{\prime}$ larger than (26), over the tuning $\lambda_{j}$ 's and $Q_{j}$ 's but not arbitrarily, due to its first term. It confirms, despite having unstable plants and sparse capacity which might cause a subsystem with large error wait for transmission, the expected error remains bounded. Moreover, (26) is derived considering the couplings between the subsystems which occurs in the communication channel.

It is shown earlier that condition (16) holds at time $k+N$ within each case $c_{1}-c_{2}$ and $l_{1}^{c_{3}}$, which implies that they stay bounded in expectation over longer finite horizons. Thus, rewriting (19) over the extended interval $\left[k, k_{m}\right]$ yields

$$
\begin{aligned}
& \sum_{i=1}^{N+m} \mathrm{E}\left[\left\|e_{k_{m}}^{i}\right\|_{Q_{i}}^{2}\right]=\sum_{c_{l}} \mathrm{P}_{c_{l}} \mathrm{E}\left[\left\|e_{k_{m}}^{i}\right\|_{Q_{i}}^{2} \mid c_{l}, \mathrm{P}_{c_{l}}\right] \\
& \leq \sum_{c_{1}} \mathrm{E}\left[\left\|e_{k_{m}}^{i}\right\|_{Q_{i}}^{2} \mid c_{1}\right]+\sum_{c_{2}} \mathrm{E}\left[\left\|e_{k_{m}}^{i}\right\|_{Q_{i}}^{2} \mid c_{2}\right] \\
& +\sum_{l_{1}^{c_{3}}} \mathrm{E}\left[\left\|e_{k_{m}}^{i}\right\|_{Q_{i}}^{2} \mid l_{1}^{c_{3}}\right]+\sum_{l_{2}^{c_{3}}} \mathrm{P}_{l_{2}}^{c_{3}} \mathrm{E}\left[\left\|e_{k_{m}}^{i}\right\|_{Q_{i}}^{2} \mid l_{2}^{c_{3}}, \mathrm{P}_{l_{2}}^{c_{3}}\right]<\bar{\varsigma},
\end{aligned}
$$

where $\bar{\varsigma}$ sums up the finite uniform upper bounds for cases $c_{1}-c_{3}$, assuming at least one successful transmission over $\left[k, k_{m}\right]$. This ensures the error Markov chain $e_{k}$ satisfies (16), which in turn affirms the overall NCS possesses LSP.

The second collision scenario prevents employing the probability $\mathrm{P}_{l_{2}^{c_{3}}}$ to show (16) holds for sub-case $l_{2}^{c_{3}}$, since no transmission happens over $[k, k+N]$. To infer (17), we calculate the probability that at least two subsystems select identical waiting times at every time step, leading to an alltime-step collision scenario. As all subsystems operate in open-loop, we calculate (17) for all $i \in\{1, \ldots, N\}$. Assume a subsystem $i$ has selected $\nu_{k^{\prime}}^{i}=s_{k^{\prime}}^{i} \tau$ at some time $k^{\prime}$, for $s_{k^{\prime}}^{i} \in\{1, \ldots, h-1\}$. The probability that a subsystem $j$ has identical waiting time $\nu_{k^{\prime}}^{j}=s_{k^{\prime}}^{i} \tau$ can be calculated. We know

$$
\mathrm{E}\left[\nu_{k^{\prime}}^{j}\right]=\sum_{m=1}^{h-1} m \tau \cdot \mathrm{P}\left(\nu_{k^{\prime}}^{j}=m \tau\right)
$$

Therefore, we conclude

$$
\begin{gathered}
\mathrm{P}\left(\nu_{k^{\prime}}^{j}=s_{k^{\prime}}^{i} \tau\right)=\frac{1}{s_{k^{\prime}}^{i} \tau}\left[\mathrm{E}\left[\nu_{k^{\prime}}^{j}\right]-\sum_{m=1, m \neq s_{k^{\prime}}^{i}}^{h-1} m \tau . \mathrm{P}\left(\nu_{k^{\prime}}^{j}=m \tau\right)\right] \\
<\frac{1}{s_{k^{\prime}}^{i} \tau}\left[\frac{1}{\lambda_{j}}-\sum_{m=1, m \neq s_{k^{\prime}}^{i}}^{h-1} m \tau . \mathrm{P}\left(\nu_{k^{\prime}}^{j}=m \tau\right)\right] \leq \frac{1}{s_{k^{\prime}}^{i} \tau \lambda_{j}} .
\end{gathered}
$$

Extending this for every pair of subsystems $i$ and $j$ which collides at every time step $k^{\prime} \in[k, k+N]$, we find the upper bound for the probability of having successive collisions as

$$
\mathrm{P}\left[\sum_{i=1}^{N} \theta_{k^{\prime}}^{i}=0, \forall k^{\prime} \in[k, k+N]\right] \leq \prod_{k^{\prime}=k}^{k+N} \sum_{i=1}^{N} \sum_{j=1, j \neq i}^{N} \frac{1}{s_{k^{\prime}}^{i} \tau \lambda_{j}} .
$$

From (21), if no subsystem transmits we can choose $\bar{\xi}^{\prime}=$ $\sum_{i=1}^{N}\left\|A_{i}^{N} e_{k}^{i}+\sum_{r=1}^{N} A_{i}^{N-r} w_{k+r-1}^{i}\right\|_{Q_{i}}^{2}>0$. Then, we have

$$
\sup _{e_{k}} \mathrm{P}\left[\sum_{i=1}^{N}\left\|e_{k+N}^{i}\right\|_{Q_{i}}^{2} \geq \bar{\xi}^{\prime}\right]<\prod_{k^{\prime}=k}^{k+N} \sum_{i=1}^{N} \sum_{j=1, j \neq i}^{N} \frac{1}{s_{k^{\prime}}^{i} \tau \lambda_{j}}
$$

for an arbitrary $\rho\left(\bar{\xi}^{\prime}, \bar{\xi}\right)$ such that $\sum_{i=1}^{N}\left\|e_{k}^{i}\right\|_{Q_{i}}^{2}<\rho$ and LSP of the overall NCS is readily obtained according to (17).

Remark 2: One can infer from (27) that, by having extremely large thresholds $\lambda_{i}$ or infinite number of communication samples $h$, the probability of having consecutive collisions converges to zero. This is expected as by enlarging the error thresholds, we are keeping more subsystems out of the access competition. In addition, as $h \rightarrow \infty$, the chance of collisions converges to zero. Note that, excluding the second scenario, the stronger stability notion, in terms of Lyapunov mean square stability (LMSS), will be obtained [16].

Remark 3: As the number of subsystems occupying the communication channel increases, the probability of collisions grows. Nevertheless, we still can show LSP as long as the ratio $h / N$ is larger than zero, i.e. $N$ is finite. It is to be expected, however, that the performance of the overall system decreases as the ratio $h / N$ decreases. A More detailed performance analysis is subject to future work.

\section{NUMERICAL RESULTS}

In this section, the performance of the proposed decentralized scheduler is compared with TDMA and idealized CSMA protocols. Furthermore, we compare our results with those obtained from the centralized and pure probabilistic scheduling policies introduced in [13] and [18], respectively.

Consider an NCS comprised of two heterogeneous classes of subsystems, one includes homogeneous control loops with unstable plants while the other contains homogeneous loops with stable processes. The system parameters are $A_{1}=1.25$, $B_{1}=1$ for the stable class, and $A_{2}=0.75, B_{2}=1$ for the unstable class. The initial state is $x_{0}^{1}=x_{0}^{2}=0$, and the disturbance is given by $w_{k}^{i} \sim \mathcal{N}(0,1)$. We consider $N$ individual loops with equal number of loops belonging to each class. To stabilize the subsystems, we choose a deadbeat control law $L_{i}=A_{i}$, and we select $Q_{i}=I$ for both classes.

Fig. 3 provides a comparison of aggregate error variance between our proposed policy and the related protocols for NCSs with different number of subsystems $N \in$ 


\begin{tabular}{|l||c|c|c|c|c|}
\hline Number of plants $(N)$ & 2 & 4 & 6 & 8 & 10 \\
\hline Error threshold $(\lambda)$ & 0.25 & 1.45 & 2.80 & 4.30 & 6.00 \\
\hline Collisions in $2 \times 10^{5}$ samples & 252 & 972 & 2094 & 3535 & 5009 \\
\hline Collisions $(\%)$ & 0.126 & 0.486 & 1.047 & 1.767 & 2.504 \\
\hline
\end{tabular}

TABLE I

SELECTED ERROR THRESHOLDS AND THE NUMBER OF COLLISIONS.

$\{2,4,6,8,10\}$ subject to the constraint (7). Note that for $N>2$, we have more unstable systems than the available transmission slots, which ensures at least one unstable system operates in open-loop at each time step. TDMA is a timetriggered access scheme, where subsystems transmit periodically. As for TDMA simulation results, we consider periodic transmissions for each loop with periods of exactly $N$ time steps. ICSMA operates statically such that the chance of transmission is $\frac{1}{N}$ for each subsystem at each time step. The averages are calculated via Monte Carlo simulations over a horizon of $2 \times 10^{5}$ samples. For comparison we also consider the case where all subsystems transmit in every time step by relaxing the capacity constraint to $c=N$, i.e. every subsystem transmits at every time step. This is the lower bound for error variance. For simplicity, we calculate the error variances by considering equal local error thresholds $\lambda$ for all subsystems in an NCS, according to Table I. The sampling rate of the communication channel is $h=150$. The number of detected collisions is shown in Table I. The waiting times are chosen randomly from the Poisson distributions for each subsystem at every time-step.

The ICSMA protocol results in an acceptable performance only for $N=2$, while the bi-character approach outperforms TDMA. The deterministic feature of our policy can be removed by setting the error thresholds to zero to obtain a pure probabilistic scheduler which considers chances for all subsystems for transmission at every time-step [18]. As expected, the error variance increases compared to the bicharacter scheduler, since those subsystems with small errors now have non-zero chances to utilize the channel.

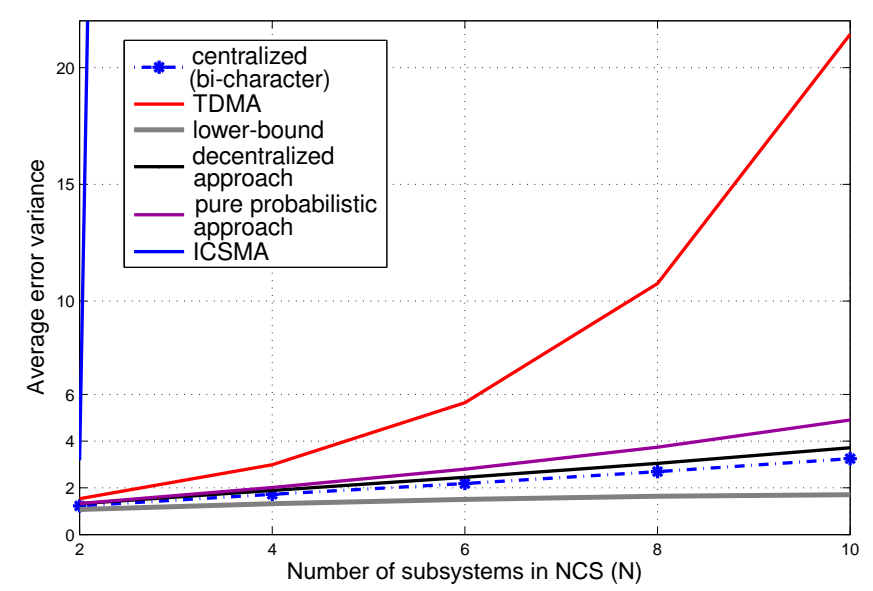

Fig. 3. Comparison of the average error variance vs. the number of control loops for different scheduling policies.

\section{CONCLUSIONS}

We propose a decentralized scheduling mechanism for NCSs over shared communication mediums. Given stabilizing control laws, stability of the overall multi-loop NCS in terms of Lyapunov stability in probability is studied. Our scheme is locally implemented and combines features of both deterministic and probabilistic designs. The deterministic feature helps to efficiently allocate the channel to the subsystems with large errors, and the probabilistic character facilitates prioritization and accounts for collisions.

\section{REFERENCES}

[1] R. Murray, K. Astrom, S. Boyd, R. Brockett, and G. Stein, "Future directions in control in an information-rich world," Control Systems, IEEE, vol. 23, pp. 20-33, Apr 2003.

[2] W. P. M. H. Heemels, J. H. Sandee, and P. P. J. Van Den Bosch, "Analysis of event-driven controllers for linear systems," International Journal of Control, vol. 81, no. 4, pp. 571-590, 2008.

[3] P. Tabuada, "Event-triggered real-time scheduling of stabilizing control tasks," Automatic Control, IEEE Transactions on, vol. 52, no. 9, pp. $1680-1685,2007$.

[4] D. Dimarogonas and K. Johansson, "Event-triggered control for multiagent systems," in Decision and Control, 2009 held jointly with the 2009 28th Chinese Control Conference. CDC/CCC 2009. Proceedings of the 48th IEEE Conference on, pp. 7131-7136, Dec 2009.

[5] D. Tolić and R. Fierro, "Decentralized output synchronization of heterogeneous linear systems with fixed and switching topology via selftriggered communication," in American Control Conference, pp. 46554660, June 2013.

[6] A. Molin and S. Hirche, "On the optimality of certainty equivalence for event-triggered control systems," Automatic Control, IEEE Transactions on, vol. 58, no. 2, pp. $470-474,2013$.

[7] A. Molin and S. Hirche, "A bi-level approach for the design of eventtriggered control systems over a shared network," Discrete Event Dynamic Systems, vol. 24, no. 2, pp. 153-171, 2014.

[8] D. Nesic and A. Teel, "Input-output stability properties of networked control systems," Automatic Control, IEEE Transactions on, vol. 49, no. 10, pp. 1650-1667, 2004.

[9] G. C. Walsh, H. Ye, and L. G. Bushnell, "Stability analysis of networked control systems," Control Systems Technology, IEEE Transactions on, vol. 10, no. 3, pp. 438-446, 2002.

[10] C. Ramesh, H. Sandberg, and K. Johansson, "Stability analysis of multiple state-based schedulers with CSMA," in Decision and Control (CDC), 2012 IEEE 51st Annual Conference on, pp. 7205-7211, 2012.

[11] M. Donkers, W. Heemels, D. Bernardini, A. Bemporad, and V. Shneer, "Stability analysis of stochastic networked control systems," Automatica, vol. 48, no. 5, pp. 917-925, 2012.

[12] M. Tabbara and D. Nesic, "Input-output stability of networked control systems with stochastic protocols and channels," Automatic Control, IEEE Transactions on, vol. 53, no. 5, pp. 1160-1175, 2008.

[13] M. Mamduhi, A. Molin, and S. Hirche, "Event-based scheduling of multi-loop stochastic systems over shared communication channels," in 21st International Symposium on Mathematical Theory of Networks and Systems (MTNS), pp. 266-273, Jul 2014.

[14] D. Christmann, R. Gotzhein, S. Siegmund, and F. Wirth, "Realization of Try-Once-Discard in wireless multihop networks," IEEE Transactions on Industrial Informatics, vol. 10, pp. 17-26, Feb 2014.

[15] A. Molin and S. Hirche, "Price-based adaptive scheduling in multiloop control systems with resource constraints," in Price-based Adaptive Scheduling in Multi-Loop Control Systems with Resource Constraints (I. T. Automatic Control, ed.), pp. 3282 - 3295, 2014. Special Issue on Control of Cyber-Physical Systems.

[16] F. Kozin, "A survey of stability of stochastic systems," Automatica, vol. 5, pp. 95-112, Jan. 1969.

[17] S. P. Meyn and R. Tweedie, "State-dependent criteria for convergence of markov chains," The Annals of Applied Probability, pp. 149-168, 1994.

[18] M. Mamduhi, A. Molin, and S. Hirche, "Stability analysis of stochastic prioritized dynamic scheduling for resource-aware heterogeneous multi-loop control systems," in Decision and Control (CDC), 2013 IEEE 52nd Annual Conference on, pp. 7390-7396, Jan 2013. 